\title{
La théologie politique des dévots Mathieu de Morgues et Michel de Marillac, opposants au cardinal de Richelieu
}

\author{
CAROLINE MAILLET-RAO
}

University of Alberta

The position of Chief Minister established by Cardinal Richelieu conjoined the minister's role with the exercise of power. Since all final decisions remained with the king, this system of government was claimed as legitimate. Nevertheless, there were opponents of Richelieu's ministry. The devout faction, represented by Mathieu de Morgues et Michel de Marillac, saw the new institution as a change to the current theory of monarchy, and thus open to legal challenge. On that basis, they laid out their opposition with a set of arguments. This essay analyzes the arguments, against Richelieu's ministry, that refer to the principles of monarchy.

L e début du XVII ${ }^{\mathrm{e}}$ siècle apparaît comme le temps où s'est affirmé la nécessité de mettre en œuvre le pouvoir absolu, afin de préserver l'obéissance au roi. En effet, les désordres politico-religieux de la seconde moitié du XVI ${ }^{\mathrm{e}}$ siècle ont amené la conviction, chez les contemporains, de l'obligation de restaurer l'autorité du roi et de renforcer encore l'absolutisme.

Dans le cadre de ce contexte historico-politique, le règne de Louis XIII a été marqué plus particulièrement par le ministériat du cardinal de Richelieu, mais aussi par une lutte politique féroce à laquelle se sont livrés Richelieu et le parti dévot $^{1}$, au sein du conseil du roi. Le parti dévot était réuni autour de la reine mère Marie de Médicis (1573-1642). Ses principaux représentants étaient Michel de Marillac (1560-1632) et Mathieu de Morgues (1582-1670). Michel de Marillac a pris officiellement la tête du parti dévot en 1629. Personnage important du règne de Louis $\mathrm{XIII}^{2}$, il a été garde des sceaux de 1626 à 1630. Ses idées se retrouvent dans ses écrits officiels, notamment la grande ordonnance réformatrice de janvier $1629^{3}$, dans sa correspondance avec le cardinal de Richelieu, dans un discours sur le bon gouvernement ${ }^{4}$, et dans divers mémoires ${ }^{5}$. Prédicateur ordinaire de la reine mère, Mathieu de Morgues défendait le parti dévot des attaques des écrivains cardinalistes. Il a justifié les positions politiques du parti dévot dans des pamphlets, écrits à la hâte, pas très bien structurés mais, néanmoins, cohérents du point de vue du contenu. 
Bien qu'elle n'ait pas été oubliée, la lutte entre le parti dévot et Richelieu a été négligée par l'historiographie, en partie parce que Richelieu a tout fait pour faire passer ses adversaires pour des personnages de moindre importance sur le plan des idées. Des Mémoires du cardinal vient l'idée, longtemps véhiculée par l'historiographie ${ }^{6}$, que l'opposition des dévots à Richelieu était une opposition à l'absolutisme que le cardinal a contribué à mettre en œuvre sous le règne de Louis XIII. Les dévots auraient donc soutenu la monarchie traditionnelle, plutôt que la monarchie absolue. Or, cette thèse est sur le point d'être remise en question ${ }^{7}$; on considère maintenant l'hypothèse que les dévots étaient eux-mêmes absolutistes et que c'est justement pour cette raison qu'ils se sont opposés à Richelieu. En effet, s'il n'est pas contestable que Richelieu ait contribué à affermir le pouvoir absolu en France, le cardinal a aussi tenté de se l'approprier officiellement, en proposant l'intégration d'une nouvelle institution dans la constitution monarchique : celle de principal ministre. Dans la pensée de Richelieu, cette institution est définie comme la délégation par le roi de l'exercice du pouvoir royal à un principal ministre, dans le cas où le roi ne voudrait pas l'exercer lui-même. C'est cette nouvelle institution que les dévots ont dénoncée comme une usurpation du pouvoir royal et une déformation de la constitution monarchique.

Le ministériat est fondé sur une théologie politique, c'est-à-dire sur des croyances religieuses justifiant tel régime politique, que l'on pourrait croire conforme à celle de la monarchie absolue ; or, ce qui est intéressant dans la critique du ministériat par les dévots, c'est qu'elle fait ressortir à quel point la théologie politique du cardinal de Richelieu était originale et juridiquement contestable. La critique du ministériat par les dévots s'est développée à l'aide de toutes sortes d'arguments. Toutefois, leur argument principal qui conteste le caractère légitime du ministériat prend racine dans une autre théologie politique, que les dévots considèrent être celle de la monarchie absolue. Finalement, les dévots opposent une autre théologie politique à celle du cardinal de Richelieu fondant le ministériat, à savoir celle de la monarchie absolue. Ils démontrent ainsi la non-conformité du ministériat à la théorie de la monarchie absolue.

\section{La non-conformité du ministériat à la théorie de la monarchie absolue}

Dans cette première partie, nous étudierons tour à tour les points de vue de Mathieu de Morgues et de Michel de Marillac. Nous avons choisi d'étudier la pensée de Morgues en premier lieu, et ce, même si du point de vue chronologique, il aurait été plus logique de traiter d'abord de celle de Michel de Marillac ; Mahieu de Morgues 
étant mort après Marillac. Néanmoins, la pensée de Mathieu de Morgues, plus élaborée, éclaire celle de Marillac. Cela permet aussi d'établir les liens clairement.

\section{Dans la pensée de Mathieu de Morgues}

L'histoire se souvient de Mathieu de Morgues pour avoir été l'un des adversaires les plus acharnés du cardinal-ministre Richelieu. La postérité a essentiellement retenu la virulence de ces propos, ce qui les qualifie effectivement. Morgues accusait le cardinal de tyrannie, tel qu'on l'observe dans cet extrait :

«L'ambition étant arrivée au sommet de ses prétentions, se change en présomption, audace, témérité, cruauté, et tyrannie : [ ... ] elle se plaît à faire sentir son pouvoir aux plus grands, et à donner l'épouvante aux plus petits craignant tout le monde, elle se fait craindre à tous, et ne pouvant se faire aimer de personne, se rend redoutable à chacun. Elle se fait environner de gardes, non pas comme un roi, mais comme un tyran $\gg^{8}$.

En particulier, Mathieu de Morgues n'acceptait pas que les écrivains du cardinal écrivent que les favoris exercent le gouvernement de l'État, ou bien que la direction de l'État appartient au principal ministre : «J'en trouve un bien plus grand $<$ blasphème > en ce discours : les favoris du roi qui gouvernaient l'État sous le nom de S.M. $\gg 9$, et :

« Sa conclusion <de Dupleix > est : [ ... ] il fut élu le premier ministre d'Estat par S.M. à Compiègne, avec tant d'avantages qu'elle lui remit en main toute la direction de son Estat. Sauf correction de M.l'historien, dans ce discours il n'y a rien de véritable que la louange du roi, qui est accompagnée d'un horrible blasphème contre sa personne sacrée $[\ldots]$ On arrache le sceptre de la main de S.M. par ces paroles horribles que : le roi a remis en la main du cardinal toute la direction de son royaume. N'est-ce pas, écrire, en termes, qu'il semble que le roi soit devenu mineur ou faible d'esprit et que d'un conseiller, il ait fait un tuteur ou un régent [ ... ] Personne ne se peut imaginer hors du cardinal de Richelieu, qui se dit directeur général du royaume, que quelqu'un (sans se rendre criminel de lèse-majesté) puisse aspirer au gouvernement qui appartient au roi seul. L'historien a très mal parlé usant du mot : commandement, au lieu de celui de crédit ou faveur $[\ldots]$ Ce $[\ldots]$ royaume est aujourd'hui en un état par les mauvais conseils du cardinal de Richelieu, que personne ne songe ni à ses vengeances ni à ses affaires particulières, parce qu'on craint la chute de la monarchie $\gg^{10}$.

Ces critiques sont-elles vides de sens et purement gratuites comme il est communément admis ou, au contraire, relèvent-elles d'une véritable position ou opposition politique ? En réalité, la dénonciation de la tyrannie de Richelieu par 
de Morgues apparaît comme une aversion profonde pour l'association d'un favori au gouvernement ${ }^{11}$. Cette association a été établie dans le système monarchique par Richelieu sous l'appellation de $\ll$ ministériat $»^{12}$. L'on entend par ministériat le «système de gouvernement qui faisait régner le roi par l'intermédiaire d'un premier ministre $\gg^{13}$. Mathieu de Morgues critiquait vivement le fait que dans le système du ministériat, toute la direction du royaume était remise au principal ministre. Cette critique n'avait rien d'abusif, car c'est exactement comme tel que Richelieu l'envisageait lui-même. En effet, Richelieu considérait que le roi pouvait se décharger du gouvernement de son royaume sur un principal ministre, ce dernier jouissant alors théoriquement des pouvoirs les plus étendus ${ }^{14}$ qui dépassaient le simple conseil du roi. Ce faisant, Richelieu conférait à cette délégation un fondement quasi-divin : « S’il est vrai que le gouvernement monarchique imite plus celui de Dieu qu'aucun autre, si le souverain peut ou ne veut pas lui-même avoir l'œil sur sa carte et sa boussole, la raison veut qu'il en donne particulièrement la charge à quelqu'un par-dessus tous les autres $\gg^{15}$. En établissant un rapport analogique entre les gouvernements divin et humain, et en invoquant, simultanément, la raison, qui est, au XVII ${ }^{\mathrm{e}}$ siècle, le chemin qu'emprunte la volonté de $\mathrm{Dieu}^{16}$, Richelieu donnait à sa théorie, comme à sa fonction, un fondement divin. À ce propos, nombre d'historiens ont souligné le fait que Richelieu se considérait comme l'instrument de Dieu ${ }^{17}$. Ainsi, le principal ministre exerçait bien des fonctions relevant du pouvoir souverain du roi. Selon cette configuration, le bonheur de l'État ne dépendait plus du bon gouvernement du roi mais de celui de son principal ministre : «Le prince doit connaître par lui-même celui qu'il chargera d'un si grand emploi [ ... ] il est vrai de dire que le bonheur ou le malheur des Estats dépend de l'élection qui en sera faite, ce qui oblige étroitement les souverains ou à prendre eux-mêmes le soin dont le poids de leur couronne les charge, ou à si bien choisir celui sur lequel il voudrait s'en décharger que leur action soit approuvée du ciel et de la terre $\gg^{18}$.

$\mathrm{Au}$ contraire, pour de Morgues, ces lignes étaient injustifiables au regard de la théorie de la monarchie. Il s'agissait d'une tentative d'usurpation du pouvoir royal, qui ne pouvait en aucun cas, même si le roi le désirait, être transmis à un principal ministre. Et en effet, Richelieu a eu des scrupules à exercer un pouvoir qu'il ne pouvait ni ne devait détenir ${ }^{19}$. Pour de Morgues, le pouvoir du roi était tout aussi indivisible qu' ill'était pour ses contemporains, comme Le Bret par exemple : «Mais l'on demande, si le roi peut faire et publier tous ces changements de lois et d'ordonnances, de sa seule autorité, sans l'avis de son Conseil ni celui de ses cours souveraines. A quoi l'on répond, que cela ne reçoit point de doute, pour ce que le roi est seul souverain dans son royaume, et que la souveraineté n'est non plus 
divisible que le point en la géométrie $\gg^{20}$. Mathieu de Morgues ne s'est donc pas opposé à la mise en œuvre de l'absolutisme par le gouvernement de Louis XIII, car le ministériat n'était pas la mise en œuvre de la monarchie absolue, et ce, même s'il est reconnu que le cardinal a tenté de mettre à exécution la théorie du pouvoir absolu $^{21}$. Aux yeux de Mathieu de Morgues, ce n'était tout simplement pas à Richelieu de le faire ni de prétendre le faire. De la même façon que l'a affirmé Jean Bodin ${ }^{22}$, la souveraineté pour de Morgues ne pouvait appartenir et n'être exercée que par le Roi. Blâmer la trop grande influence d'un favori constituait, pour de Morgues, une véritable opposition au ministériat et démontre son adhésion au gouvernement personnel du royaume par le roi ${ }^{23}$. Bien évidemment, il va de soi que Mathieu de Morgues réfléchit dans le cadre d'un ministériat bien défini comme «l'association de Richelieu à l'exercice du pouvoir aux côtés d'un autre homme par lui perçu comme élu par Dieu, le roi dont il ne faut jamais oublier qu'il ne pouvait être que le second, l'instrument intelligent de la volonté éclairée, celui qui n'œuvre que pour la gloire du roi, laquelle seule subsiste à l'heure du bilan politique $\gg^{24}$ selon la définition de Françoise Hildesheimer. Pourtant si, pour Richelieu, le fait que le roi soit le seul à prendre les décisions au final suffisait à justifier le système du ministériat, pour Mathieu de Morgues, il n'en était rien. Même si Richelieu porte tout ce qu'il fait au crédit du roi, le problème pointé par de Morgues est celui du rôle trop important de Richelieu dans le processus de décision, car le ministériat, tel qu'il est définit par Richelieu lui-même dans son Testament politique, n'est pas le gouvernement de deux associés, ni celui d'un roi conseillé par son favori, mais le gouvernement du principal ministre dont les décisions sont entérinées par le roi, celui d'un monarque « qui ne veut pas avoir lui-même l'œil sur sa carte et sa boussole ». L'idée qu'un roi déléguerait, tout en conservant bien sûr le dernier mot, le maniement des affaires et le pouvoir, au profit d'un principal ministre est tout simplement incompatible avec la conception que se fait de Morgues du gouvernement monarchique : l'exercice absolu du pouvoir par un roi de droit divin.

\section{Dans la pensée de Michel de Marillac}

Marillac a combattu le ministériat dans son Discours sur le bon gouvernement, en affirmant que le roi devait toujours conserver les rênes du pouvoir entre ses mains.

«Le roi, comme tous ceux qui commandent les grands estats, doit avoir en sa main l'autorité et la conduite de la puissance nécessaire au gouvernement de son Estat, en tenir les rênes pour les lâcher ou retirer selon les occasions, car en cela consiste le bon gouvernement. Pour ce faire, il faut qu'il sache à qui il a à faire, ce qu'il faut 
aimer et fuir en tous ceux qui dépendent de lui ou avec qui il a des affaires ce en quoi chacun d'eux peut servir, et en quoi nuire. Jusqu'où on doit laisser aller. Et quelle borne chacun doit avoir usage de la puissance qui lui est donnée. Car il ne faut laisser à personne l'usage de sa puissance entier et indéfini. Et le bon ordre du gouvernement consiste en ce que chacun demeure dans les termes du pouvoir qui lui est donné non seulement en l'étendue du pouvoir, mais aussi en la manière d'en user $\gg^{25}$.

Il n'existe pas d'information sur le lieu où a été prononcé ce discours inédit. Conservé aussi au ministère des affaires étrangères, il se trouve dans le volume 792 des mémoires et documents de la France, qui renferme des documents de l'année 1628. L'index des documents contenus dans ce volume annonce ce texte sous le titre « Assemblée du clergé, discours de M. de Marillac ». Cela laisse donc penser que ce discours a été prononcé lors de l'Assemblée du clergé qui s'est tenue en 1628. Toutefois, nous ne voyons pas pourquoi le garde des sceaux aurait prononcé un tel discours sur le bon gouvernement devant une assemblée réunie dans le but d'examiner la contribution du clergé à la réduction de la dette de l'État. Il nous semble que ce discours date plutôt du 10 juin 1630 car l'inventaire des lettres du garde des Sceaux, contenu dans le volume 249 des Mémoires et documents de la France conservé au ministère des affaires étrangères, indique un « Discours de M. le garde des Sceaux sur la manière de gouverner l'Estat en date du 10 juin $1630 \gg$. Le résumé de ce texte présent dans l'inventaire correspond exactement à texte que l'on examine : « Il veut que le Roi ne mette jamais l'autorité en un lieu qu'il tienne toujours les rennes, que chacun demeure dans les termes du pouvoir qui lui est donné $\gg^{26}$. Malheureusement, on n'y trouve aucune indication sur le contexte dans lequel a été prononcé ce discours. Le Mercure français n'apporte pas plus d'information puisqu'il ne fait aucune mention de ce discours du chancelier, et ce, malgré son importance. Il est tout à fait probable que le cardinal ait pris des mesures pour qu'il ne soit fait mention de ce discours nulle part, étant donné sa position contre le ministériat.

En juin 1630, la puissance de Richelieu était déjà extraordinaire et il en était presque fini du parti dévot, puisqu' il allait tomber cinq mois plus tard, en novembre 1630, lors de la journée des dupes. Bien sûr, le dévot Marillac est conscient de l'influence de Richelieu. Mais cela ne l'empêche pas de critiquer presque ouvertement le ministériat. Évidemment, sa position de garde des sceaux ne lui permet pas d'être aussi direct que Mathieu de Morgues et d'attaquer directement le cardinal ministre. Il n'en reste pas moins que définir le «bon gouvernement » comme celui dans lequel le roi a en sa main l'autorité et la conduite de la puissance et dire qu'il ne faut laisser à personne d'autre l'usage entier et indéfini de sa puissance constituent une attaque 
directe de la volonté de Richelieu de s'établir principal ministre avec pour charge d'exercer le pouvoir à la place du roi. D'ailleurs, à cette époque, il ne fait aucun doute que le cardinal disposait du pouvoir : c'est lui qui dirigeait les opérations militaires en Italie contre l'Espagne Habsbourgeoise, qui menait les négociations et qui finalement a entraîné la France dans ladite guerre de Trente ans. C'est bien dans ce contexte que le texte de Marillac intervient pour critiquer non pas la politique menée, mais l'homme qui dirigeait l'État sans en avoir reçu le mandat. Bien que Marillac applique à tous les ordres du royaume la maxime qu'il ne faut laisser à personne l'usage entier et indéfini de sa puissance (le clergé ${ }^{27}$; la noblesse ${ }^{28}$; le Tiers État représenté, d'abord selon Marillac, par les Parlements ${ }^{29}$, ensuite par les habitants des bonnes villes ${ }^{30}$ et, enfin, par les huguenots ${ }^{31}$ ), cela concerne d'autant plus ses ministres, et d'abord, le premier d'entre eux, le cardinal de Richelieu. D'autre part, il est intéressant de remarquer que, dans ce passage, Marillac fait appel au concept d'État pour appuyer l'indivisibilité de la souveraineté du roi. Cela est suffisamment rare pour qu'il en soit fait état. De même, à quelques occasions, Morgues a attribué la souveraineté à l'État abstrait. Plus exactement, de Morgues a recouru à la notion d'État pour contrer ceux qui prétendaient partager la souveraineté et ainsi, appuyer l'idée que le pouvoir appartenait exclusivement au roi. Lorsqu'il n'y avait pas de danger, les dévots évoquaient plutôt la «souveraineté du roi $\gg^{32}$. Ainsi, l'on peut supposer que puisque Marillac fait appel au concept d'État dans son discours, c'est qu'il estime que la souveraineté du roi est en danger. Un tel discours ne se justifierait d'ailleurs pas dans des circonstances ordinaires. Finalement, l'usage que font les dévots de l'idée d'État étaie l'idée que ce concept était utilisé pour défendre le pouvoir royal des agressions idéologiques et des révoltes.

L'étude des critiques du ministériat, développées par les dévots démontre la non-conformité de la nouvelle institution introduite par le cardinal de Richelieu. Celle-ci n'avait pas sa place dans le cadre du régime monarchique français, fondé sur l'idée que le roi était le seul habilité à exercer le pouvoir.

À cette prétention d'exercer le pouvoir royal par délégation, les dévots ont répondu en prenant appui sur les fondements idéologiques de la monarchie : le droit divin et l'idéologie paternelle.

\section{La théologie politique des dévots, conforme à celle de la monarchie absolue}

La théologie politique des dévots est, en tous points, conforme à celle de la monarchie absolue, telle qu'elle sera mise en place par les rois Louis XIV et Louis XV. 
Elle repose sur la nouvelle doctrine du droit divin, qui exclut de l'exercice de la souveraineté tous ceux qui prétendaient y participer tels que les grands officiers, les Grands ou le principal ministre.

\section{Dans la pensée de Mathieu de Morgues}

Mathieu de Morgues a critiqué la doctrine du ministériat en mettant en avant l'idée qu'elle bouleversait l'ordre monarchique. La démarche de Richelieu n'était pas de remettre en cause la conception absolue de la monarchie qu' il soutenait ; il tentait plutôt de la mettre en œuvre. Cependant, Richelieu tâchait également de faire instaurer une nouvelle institution et de lui conférer un fondement divin. Pour de Morgues, la délégation du pouvoir royal portait atteinte à la personne sacrée du roi ${ }^{33}$. La critique du ministériat de Mathieu de Morgues reposait donc sur la doctrine du droit divin. Pour de Morgues, seul le roi bénéficiait de ce privilège d'avoir été choisi par Dieu pour gouverner le royaume. Ainsi, de Morgues défendait la nouvelle doctrine du droit divin, élaborée par les Politiques Jean Bodin et Pierre de Beloy à la fin $\mathrm{du} \mathrm{XVI}^{\mathrm{e}}$ siècle, pour riposter aux théories des partis monarchomaques protestants et la Ligue catholique, qui soutenaient l'idée selon laquelle le roi tenait son pouvoir médiatement de Dieu par l'intermédiaire du peuple. Cette vision impliquait une responsabilité du roi devant son peuple. Pour les monarchomaques comme pour les ligueurs, la monarchie reposait même sur un contrat passé entre le roi et son peuple, qui faisait de ce dernier le juge du gouvernement royal. Ainsi, le peuple réuni en assemblée était en mesure de déposer un roi, voire de l'éliminer. Ces doctrines avaient conduit à l'assassinat des deux rois, Henri III en 1589, et Henri IV en 1610. Malgré ces drames, les théories monarchomaques étaient toujours véhiculées par le parti protestant. Mathieu de Morgues s'est appuyé sur la doctrine du droit divin pour les combattre dans son pamphlet, Le droit du roi sur des sujets chrétiens, à ceux de la RPR, dont voici un extrait :

« Finalement, vous ne doutez pas que vous ne soyez dans une monarchie ancienne, établie sur des lois certaines, justes et raisonnables, et qui est gouvernée par un roi, lequel ne vous a pas conquis par force pour vous posséder avec violence, mais qui nous est donné du ciel, et duquel nous pouvons assurer avec plus de raison que Tertullien ${ }^{34}$ des empereurs payens, que sa puissance et son âme viennent du même lieu, puisqu'il est, par la grâce de Dieu chrétien, et très chrétien, non pas choisi tumultueusement dans un camp par des soldats brutaux et pratique comme Théodose ${ }^{35}$ ou Martian (sic) ${ }^{36}$, ni adopté par l'artifice des femmes comme Tibère ${ }^{37}$ ou Néron ${ }^{38}$, ni esclave comme Basile ${ }^{39}$, mais donné par la nature et descendant d'une grande suite de rois $\gg 4^{\circ}$. 
Ce paragraphe de Mathieu de Morgues est intéressant parce qu'il démontre toute la difficulté d'introduire une nouvelle interprétation de la doctrine du droit divin au sein de la constitution monarchique coutumière. Le problème était que la théorie du droit divin était bien une nouvelle doctrine, fruit de la réinterprétation de l'origine divine du pouvoir, visant la mise en œuvre de l'absolutisme. Pour affirmer la légitimité de l'exercice absolu du pouvoir, de Morgues choisit d'ancrer son origine dans l'histoire romaine, source de la monarchie française et qui a vu naître le christianisme. La première des sources à laquelle de Morgues se réfère pour justifier l'origine divine du pouvoir royal, est une citation du penseur et père de l'Église, Tertullien; citation qui renvoie à la réponse du Christ faite à Pilate : « Tu n'aurais aucun pouvoir sur moi, si cela ne t'avait été donné d'en haut $\gg^{41}$. Cette fameuse parole de Jésus fondait l'ancienne conception de l'origine divine d'un pouvoir qui vient d'en haut médiatement, mais sert aussi de source à sa réinterprétation qui fait venir le pouvoir souverain de Dieu immédiatement cette fois. Ainsi, les deux versions se trouvent mêlées ou entremêlées, et la plus récente, inscrite dans le temps. Elle tient là son fondement, non seulement théorique, mais historique, ce qui est primordial dans une société où l'adoption des lois fondamentales relevait de la coutume.

Ensuite, pour mieux définir cette nouvelle conception de l'origine divine du pouvoir et la distinguer de la théorie du contrat, de Morgues recourt à des exemples d'empereurs romains qu'ils aient été chrétiens ou non. Ce dernier critère n'a en fait pas d'importance pour de Morgues qui s'intéresse uniquement au mode de transmission du pouvoir. La continuité dynastique entre les empereurs était assurée selon les modes de l'adoption et de l'élection combinées. Ces moyens contrastent, évidemment, avec les règles de transmission du pouvoir en France. Plutôt que de les rappeler, de Morgues préfère recourir aux exemples romains qui, sans aucun doute, accentue la différence et démontre que la monarchie française n'était pas élective. La théorie du contrat, élaborée par les monarchomaques protestants et à la base de l'existence du parti protestant, n'est donc pas recevable. N'ayant plus de légitimité, ce dernier doit disparaître comme il en avait été décidé dans l'Édit de Nantes.

En conséquence, il est très clair que de Morgues considérait le droit de résistance allégué par le parti protestant comme irrecevable dans le cadre de la monarchie française de droit divin. Mathieu de Morgues recourait à l'enseignement spécifique des disciples du Christ plaidant en faveur de l'obéissance des chrétiens à l'empereur. Pour combattre l'idée du droit de résistance, de Morgues renvoyait son lecteur au modèle de la monarchie française qui n'est autre que celui proposé par le Christ et enseigné par les apôtres : un roi de droit divin et des sujets obéissants. La monarchie française est elle-même fidèle au modèle proposé par Jésus-Christ, à la conception 
originelle et chrétienne de la monarchie sur laquelle elle repose. Ce modèle adopté depuis ces temps immémoriaux est de ce fait immuable. Les apôtres eux-mêmes et les premiers chrétiens ont d'ailleurs obéit à cet enseignement, si l'on se réfère à l'histoire des martyrs, tels que Pierre et Paul. Du reste, de Morgues en fait également un argument contre l'idée du droit de résistance :

« Le concile de Gangtes [tenu en l'an 361] assemblé en ce siècle, et composé d'un grand nombre de Pères Grecs et Latins a frappé d'anathème et malédiction tous ceux qui assurent que les serviteurs fidèles ne doivent plus obéir aux infidèles et sont obligés en conscience de se retirer de leur service, ne dirons nous pas que l'obligation que le sujet a de se soumettre à son prince naturel et légitime est d'autant plus grande qu'elle ne vient pas de la volonté libre comme celle du serviteur qui se donne à certaines conditions et pour un temps mais qui nous est imposée de Dieu, de la nature, du droit des gens, sans exception et pour toujours. Saint Ambroise a reconnu cette vérité lorsqu'il a commandé qu'on rendit l'honneur et le tribut qu'on devait à Justine qui le persécutait et voulait détruire l'Église. Lui et Babilas se sont servis du glaive de l'excommunication pour retrancher des assemblées de l'Église les Empereurs quant ils ont donné juste sujet, mais je ne lis pas qu' ils aient jamais soulevé les peuples, ils les ont plutôt apaisé lorsque le zèle de la religion le poussait jusqu'à vouloir défendre avec les armes la vie, et la liberté de leurs Evêques $\gg 4^{2}$.

D’après de Morgues, l'obligation d'obéissance à l'égard des autorités temporelles, instituée par le Christ et enseignée par les apôtres, a été entérinée par l'Église elle-même lors du concile de Gangtes. Il devait certainement s'agir d'un concile particulier, car il ne figure pas dans la liste des conciles œcuméniques. D'après ce que Mathieu de Morgues rapporte de ce concile, l'obligation de se soumettre aux autorités politiques est un commandement divin, qui s'est ensuite inscrit dans l'histoire par le biais des premiers chrétiens dans le cadre de l'Empire romain, dont est directement issue la monarchie française. Nous pouvons également remarquer que cette vision de l'obéissance est identique à celle incluse dans la monarchie absolue, et que l'on peut observer dans la réponse que Jacques-Bénigne Bossuet fit plus tard au pasteur Jurieu qui voulait limiter le pouvoir de Louis XIV ${ }^{43}$ : «On doit obéir au prince par principe de religion et de conscience. [ ... ] Le service de Dieu et le respect pour les rois sont choses unies. C'est donc l'esprit du christianisme de faire respecter les rois avec une espèce de religion $\gg 44$. L'obéissance, chez de Morgues comme chez Bossuet, relève d'un commandement religieux et est, de ce fait, indiscutable. La comparaison nous confirme également la conformité de la conception de Mathieu de Morgues à l'esprit de l'absolutisme.

Bien entendu, Richelieu n'a pas, comme les monarchomaques protestants, prôné le tyrannicide. Il s'agissait pour lui, d'établir le pouvoir absolu. Cependant, 
Richelieu et ses écrivains avaient tendance à insister sur la puissance dont devait faire preuve le roi pour se faire obéir ${ }^{45}$. La puissance devenait alors un fondement de la monarchie absolue. De Morgues n'était pas d'accord avec ce point de vue. C'est pourquoi, il a opposé à l'« étatiste » des Montagnes ${ }^{46}$, le fondement religieux de l'obéissance des sujets au roi, qui découlait du droit divin : « Au reste, vous apprendrez, quand il vous plaira, à parler mieux que vous ne faites de la royauté : vous n'établissez la grandeur que dans l'opinion et appréhension de la seule puissance $\gg 47$. Pour de Morgues, le roi devait être obéi par ses sujets non d'après sa puissance, mais suivant le commandement de Dieu :

«Vous aurez agréable que je vous avertisse que sous le nom de la défense du roi vous le trahissez, lorsque vous n'affermissez son autorité que par l'opinion, non par la raison parmi les hommes et dans la parole de Dieu entre les chrétiens $\gg 48$.

Cette idée a contribué à fonder la mauvaise interprétation qui a été développée de la pensée des dévots. En effet, en présentant la pensée de des Montagnes comme fondée sur un élément laïque, de Morgues fait passer ce partisan de Richelieu pour machiavélien.

Cependant, il existe une réelle possibilité de se méprendre sur la pensée, quelquefois ambiguë, de Richelieu. D’une part, dans son Testament politique, il estimait que la raison obligeait l'homme « à faire que tous ceux qui sont sous son autoritéla révèrent et la suivent religieusement $\gg 49$. D'autre part, Richelieu soutenait également que le roi devait être puissant pour être respecté par ses sujets ${ }^{50}$. Néanmoins, cela ne signifie en aucun cas que l'obéissance soit devenue la nouvelle religion ${ }^{51}$. En réalité, le véritable désaccord entre les dévots et les étatistes ne résidait pas tant dans le fondement religieux de l'obéissance, que dans la connaissance de la volonté de Dieu. Ce désaccord renvoie à une dispute théologique plus générale sur la notion de raison ${ }^{52}$. À ce propos, la mention de la « raison parmi les hommes » dans la dernière citation de Morgues n'est pas innocente. Pour celui-ci comme pour les dévots, la volonté divine ne pouvait pas être connue par la raison humaine ${ }^{53}$; elle se trouvait dans « les commandements de Dieu » auxquels l'auteur fait souvent référence, ainsi que dans les signes ${ }^{54}$ et les manifestations divines ${ }^{55}$. De même, dans ce dernier passage, de Morgues considérait qu'il fallait suivre la parole divine et que la raison relevait des hommes. Au contraire, pour Richelieu, la raison était véritablement l'instrument de Dieu, destinée à guider les hommes et, dans ce cas, le moyen pour les sujets de savoir qu'il faut obéir religieusement au roi. La raison chez le cardinal ne reliait pas seulement les hommes à Dieu ${ }^{56}$; elle était le guide le plus sûr de la conduite chrétienne57, l'évidence de ce qui est juste, de ce qui est bon, 
de ce qu'il convient de faire ${ }^{58}$. Autant dans les écrits théologiques que politiques de Richelieu ${ }^{59}$, la raison était la lumière naturelle :

« La lumière naturelle fait connaître à un chacun que, l'homme ayant été fait raisonnable, il ne doit rien faire que par raison, puisque autrement il ferait contre sa nature, et, par conséquent, contre celui même qui en est l'auteur. Elle enseigne encore que, plus un homme est grand et élevé, plus il doit faire état de ce privilège, et que moins il doit abuser du raisonnement qui constitue son être, parce que l'avantage qu'il a sur les autres hommes contraint à conserver ce qui est de la nature et ce qui est de la fin que celui dont il tire son élévation s'est proposé. De ces deux principes il s'ensuit clairement que l'homme doit souverainement faire régner la raison, ce qui ne requiert pas seulement qu'il ne fasse rien sans elle ${ }^{60}$.

Dans ce sens, on pourrait se demander si René Descartes (1596-1650) n’a pas influencé la conception du cardinal de Richelieu. En effet, Descartes était connu des cercles du pouvoir puisqu'il avait attiré « la bienveillante sympathie » du dévot Pierre de Bérulle (1575-1629) par son projet de démontrer l'existence de Dieu ${ }^{61}$. De plus, des philosophes ont mis à jour la dimension religieuse de la conception cartésienne de la raison ${ }^{62}$, notamment en s'appuyant sur ce passage des Méditations métaphysiques :

«Il n'y a que la volonté [ ... ] c'est elle qui me fait connaître que je porte l'image et la ressemblance de Dieu [ ... ] Dieu dispose ainsil'intérieur de ma pensée $[\ldots$ car ne concevant rien que par le moyen de cette puissance que Dieu m’a donnée pour concevoir, sans doute que tout ce que je conçois, je le conçois comme il faut et il n'est pas possible que je me trompe $\gg^{63}$.

Les dévots fondaient doncl'obéissance des sujets au roi sur le commandement de Dieu, alors que Richelieu pensait que ce commandement était inscrit par Dieu dans la raison humaine. Finalement, de Morgues a accusé le parti étatiste de machiavélisme alors qu'il ne l'était pas, pour mieux faire ressortir le fondement religieux de l'obéissance des sujets envers leur roi de droit divin. Mathieu de Morgues s'est donc indirectement servi de la doctrine du droit divin pour critiquer le ministériat.

\section{Dans la pensée de Michel de Marillac}

Michel de Marillac a critiqué le ministériat en mettant en avant le fait que le roi ne devait « laisser à personne l'usage de sa puissance entier et indéfini $\gg^{64}$. Ainsi, «le bon ordre de gouvernement » consistait en ce que chacun demeure dans les limites du pouvoir qui lui était donné. À la lecture de ces lignes, la question qui vient à l'esprit est : à quel ordre se réfère Marillac dans l'expression « le bon ordre 
de gouvernement $\gg$ ? Et, puisque chacun doit demeurer dans les limites du pouvoir qui lui est donné, qui distribue le pouvoir?

Il va de soi que ces expressions font référence aux théories qui fondent le pouvoir. Dans le Discours sur le bon gouvernement d'où sont tirés ces extraits, Marillac n'évoque pas précisément les doctrines sur lesquelles repose la souveraineté royale. Son propos n'est pas de les rappeler, il les prend pour acquises et n'y fait qu'une référence vague, afin de justifier son opposition à tous ceux qui prétendaient partager le pouvoir du roi, dont le cardinal de Richelieu. Cependant, l'ordre que Marillac invoque dans ce discours est « l'ordre établi de Dieu en la terre ${ }^{65}$ dont il a rappelé les règles de fonctionnement aux habitants de Dijon qui s'étaient révoltés. « Cet ordre », affirme-t-il, « est dans les monarchies par l'autorité de commander et par le devoir de l'obéissance ». La souveraineté royale, tout comme l'obéissance des sujets sont de droit divin et ont Dieu pour fondement. Aux yeux de Marillac, il n'est donc pas possible d'y contrevenir. Partisan de la doctrine du droit divin, Marillacy a cependant peu fait référence. Cela s'explique par le fait qu'il était un personnage officiel du gouvernement de Louis XIII. La monarchie n'ayant pas officiellement adopté la doctrine du droit divin par crainte d'un schisme avec Rome, le garde des sceaux ne pouvait pas se permettre de la prôner.

Pour justifier le pouvoir absolu du roi, Marillac a plus souvent eu recours à l'imagerie paternelle. Ainsi, aux États généraux de Bretagne en 1626, le roi, par l'intermédiaire de son garde des sceaux Marillac, exprimait sa volonté d'être considéré comme un père : «Il veut que vous le regardiez comme père ${ }^{66}$. De même, dans son discours sur le bon gouvernement, Marillac rappelle que : « Le roi ainsi aime tous ses sujets, les conduit comme père $\gg{ }^{67}$. Lieu commun du langage politique, l'imagerie paternelle a, de tout temps, été utilisée pour désigner les rapports des rois avec leurs sujets. Les publicistes français l'ont reliée à la théorie du corps mystique. Au moment de l'accession au trône, le roi épouse le royaume : ce mariage est symbolisé par la remise de l'anneau nuptial béni lors de la cérémonie du sacre. Il ne forme plus qu'un seul corps avec son épouse mystique, le royaume, dont il est la tête, et les sujets deviennent tous ses enfants. Ainsi, à la tête du corps mystique du royaume, le roi est à la fois un chef, un époux et un père ${ }^{68}$. Si l'attribut de père est appliqué au roi depuis des temps immémoriaux, à l'époque médiévale, il ne l'était pas systématiquement. Louis XII, le roi sans héritier, se fit donner le surnom de « Père du Peuple » par l'assemblée des États généraux réunie à Plessis-lez-Tours le 14 mai 1506. Relevant par nature plus d'une assemblée de notables que de véritables États généraux ${ }^{69}$, le rôle des députés fut de témoigner de la caution de l'opinion publique au projet de mariage de la fille unique du roi, Claude, et du prince François de Valois- 
Angoulême ; et ce, pour faire face aux récriminations de la maison d'Autriche à qui la princesse avait été déjà promise. Le titre de père du peuple devint post-mortem le surnom officiel de Louis XII. Si l'on s'en tient au texte, les raisons pour lesquelles les États généraux, par la bouche de Thomas Bricot ${ }^{70}$, donnèrent à Louis XII ce surnom tiennent à l'exercice d'une politique juste et paisible, approuvée par le peuple :

«Sur quoi il vous a fait dire que ainsi qu'il a accoutumé de faire ses affaires, mêmement en ceux qui touchent le bien et utilité de son royaume et de ses sujets, lesquels il a fort à cœur, tellement que bien souvent il veille quant les autres dorment, par quoi l'avez justement baptisé Père du Peuple $\gg^{71}$.

D’un autre point de vue, Claude de Seyssel justifiait la titulature par les liens affectifs entre le roi et son peuple, et par la puissance internationale du roi de France. Alors que le rapport réciproque d'amour entre roi et père est une constante de la métaphore familiale, l'idéal impérial, pour sa part, a été complètement oublié. L'emploi de ce surnom est resté attaché au modèle d'une monarchie tempérée, où la paix était conservée, la fiscalité modérée, la justice toujours respectée et la politique conduite dans un climat d'approbation générale reflétant le lien affectif du roi avec ses sujets. Dans le cas de Louis XII, ce style de monarchie n'a pas empêché le roi de faire preuve d'autorité, et d'être prêt à utiliser une réunion d'États généraux pour renforcer l'autorité royale. L'autorité et le pouvoir de décider était propre au régime monarchique établi par le statut coutumier du royaume. Même tenu par ce statut de respecter les lois fondamentales et, notamment, les privilèges de chaque ordre, le roi, tête du corps mystique, détenait théoriquement le pouvoir absolu et bénéficiait du dernier mot. Néanmoins, dans l'exercice de son pouvoir, le roi accordait une large place à son peuple en demandant conseil aux États généraux et à ses cours suprêmes. En prenant conseil auprès de ses sujets, on avait coutume de dire que le roi gouvernait en bon père de famille, avec bienveillance gratuite.

Ayant recours à l'imagerie paternelle, les historiens ont conclu rapidement que Marillac était tenant d'une monarchie modérée, et qu'il était défavorable au climat d'autorité absolue qui était devenu nécessaire au début du XVII ${ }^{\mathrm{e}}$ siècle. Cependant, à la fin $\mathrm{du} \mathrm{XVI}$ siècle, des théoriciens recoururent au modèle patriarcal pour penser la monarchie absolue. Le roi est père de ses sujets ; il a donc l'autorité absolue du père. Le raisonnement relève également de la doctrine du droit divin : ne tenant son pouvoir que de Dieu, le roi n'en est pas comptable à ses enfants. Néanmoins, la métaphore patriarcale était aussi utilisée par les adversaires de l'absolutisme ; les parlementaires par exemple réclamaient moins d'être consultés que d'avoir un pouvoir de codécision. L'exposé d'un paternalisme traditionnel consistait à insister sur la possibilité de consulter les sujets dans le cadre de l'exercice du pouvoir législatif. 
Au contraire, Michel de Marillac affichait un paternalisme absolutiste dans le sens où il présentait la fonction du roi-père comme l'exercice d'un pouvoir absolu, en faisant l'impasse sur la consultation des sujets-enfants et la recherche du climat de consensus que cela a pu engendrer. Marillac insistait sur le caractère autoritaire du pouvoir royal, stigmatisant la monarchie absolue. Selon lui, le roi-père ne devait pas rechercher le consensus en consultant ses sujets, mais il devait maintenir toutes les parties du royaume dans les limites de leur pouvoir :

« Car il ne faut laisser à personne quelconque l'usage de sa puissance entière et indéfinie. Et le bon ordre du gouvernement consiste en ce que chacun demeure dans les termes du pouvoir qui lui est donné non seulement en l'étendue du pouvoir, mais aussi en la manière d'en user. Le roi, ainsi, aime tous ses sujets, les conduit comme père, les retient ou il faut, et les possède tous qui est son point fort nécessaire de posséder tous ses sujets bons comme mauvais $\gg^{72}$.

Ainsi, Marillac s'oppose à l'image du prince idéal incarné par Louis XII, gouvernant modérément, en accord avec ses sujets. Appliqué au premier ordre du royaume, le soin paternel consiste à contenir l'exercice du pouvoir du clergé dans ses limites. L'amour paternel même, se caractérise par une certaine réserve :

« Ce corps doit être favorisé et aimé du roi, mais comme il n’y a point de puissance en terre qui, dans la pleine liberté, ne s'étende quelquefois hors ses bornes, l'amour et l'estime du roi vers cet ordre doit être avec circonspection pour les retenir s'ils passent plus avant qu'il ne faut mais toujours avec le même amour, et le faire par soin paternel $\gg 73$.

Dans ce discours, le propos de Michel de Marillac n'est pas de démontrer que le roi doit rechercher le consensus avec ses sujets ou l'approbation de la politique royale par le premier des ordres, mais de justifier l'attitude autoritaire du roi auprès d'eux comme auprès des autres, en recourant à une imagerie paternelle au service de l'absolutisme. Ainsi, Marillac poursuit son discours en adoptant le même raisonnement à l'égard de toutes les parties du royaume. À l'égard des juridictions, qu'elles soient ecclésiastiques ou royales, Marillac affirme que le rôle du roi-père est de s'élever au-dessus de tous ses sujets-enfants afin de les contenir chacun dans l'étendue de leur pouvoir ${ }^{74}$. Quant aux parlements, Marillac ne les appuie pas, et ne pratique pas l'ambiguïté de laisser entendre qu'il serait tenant d'une monarchie modérée. Au contraire, il leur renvoie l'autorité absolue du roi :

$\ll$ Pour le regard des parlements $[\ldots$. $]$ pour ce que la grandeur de leur puissance leur laisse quelquefois oublier qu'elle ait des bornes et qu' ils entreprennent au-delà de leur pouvoir, même qu'ils s'ingèrent parfois à prendre connaissance du gouvernement de l'Estat, contrôler les rois en leur conduite et les vouloir obliger à prendre des 
conseils à leur gré. Le roi doit avec la dignité de sa couronne user de sa puissance et leur faire voir jusqu'où va le pouvoir qu'ils ont de S.M., les contenir dedans, et les empêcher de passer plus avant. Mais il ne doit pas pour cela s'offenser contre eux, mais les tenir pour ses serviteurs vers lesquels il se gouverne comme père, les retenant dans les bornes de leur pouvoir $\gg 75$.

La contribution de Michel de Marillac est intéressante en ce qu'elle permet d'illustrer la transformation de l'imagerie paternelle sous la monarchie absolue. L'amour même du roi-père s'est transformé ; il est passé de la recherche d'un consensus avec ses sujets-enfants à la tentative d'imposer des limites aux différents corps du royaume comme à tous ceux qui prétendraient prendre part à l'exercice du pouvoir souverain, y compris le principal ministre. Le roi-père ne se situe plus au-dessus de ses sujets comme le promoteur du consensus, il est l'autorité limitant chacun à son rôle. C'est ce que Marillac veut signifier quand il dit que le roi possède tous ses sujets.

Du roi-père au roi-tête du corps mystique, il n'y a qu'un pas. Marillac le prouve en faisant directement découler l'harmonie de l'État, de l'exercice de ce nouveau rôle paternel du roi : « Tous les corps sont utiles à quelque chose, et quand ils sont maintenus en leur rang, ils composent 1 'harmonie de l'Estat $\gg 7^{6}$. De même, Marillac recourt tout particulièrement à la métaphore corporelle pour expliquer comment l'amour des sujets-corps du royaume pour ce roi-père et tête du corps mystique se manifestera : par une constante obéissance et par le respect des limites que le roi leur impose. Le devoir du roi est de porter son principal soin « à ce que tous [ses] sujets vivants comme membres d'un même corps et étant unis en une même volonté, n'eussent autre but que l'obéissance envers [lui], et l'affection envers [son] Estat $\gg 77$. Il en est ainsi pour les sujets protestants,: comme pour les parlementaires réfractaires ${ }^{78}$. La grande ordonnance de janvier $1629^{79}$ avait pour finalité de réduire tous les sujets à l'obéissance. Elle réglementait les droits et les devoirs des différents corps du royaume, en commençant par ceux des Parlements. « Voulons nos dites ordonnances être observées, $[. .$.$] , sans y contrevenir [. .$.$] même sous couleur$ des dites remontrances non faites $\gg 80$.

Si Marillac prend cet exemple, ce n'est que pour appuyer le commandement divin de l'obéissance au roi $^{81}$. Le roi, évidemment, est le seul juge de la volonté de Dieu pour le royaume :

«À plus forte raison faut-il en un Estat monarchique conserver le bien et l'utilité de la monarchie et suivre l'autorité de celui qui est établi de Dieu sur nous et qui reçoit de Dieu plus que nous ni aucun de son Estat, plus immédiatement les bonnes inspirations pour le bien de l'Estat $\gg^{82}$. 
Ce pouvoir reconnu au roi d'interpréter la volonté divine est d'autant plus certain que Marillac le fait découler du droit divin ; mais c'est un autre extrait de ce passage qui attire le plus l'attention : « les bonnes inspirations ». À l'inverse de son compagnon dévot, Michel de Marillac considérerait-il que le roi puisse accéder à la volonté de Dieu par la voie de sa propre raison, par des « inspirations » dont Dieu le pénètrerait ? Cela est peu probable car, à l'exception de ce texte ci-dessus, le garde des sceaux associe souvent la volonté divine à quelque chose d'extérieur au roi : un commandement, une malédiction ou une bénédiction et la maladie :

«Mais, il faut aussi avoir grand soin que la Justice soit bien administrée, les abus réprimés, les sujets soulagés, les pauvres consolés et défendus de l'oppression des grands, car outre que la conscience du roi est déchargée, $[\ldots] \gg^{8_{3}} ; \ll$ Lequel $<$ Dieu $>$ ayant averti les rois que c'est par Lui qu'ils règnent et qu'il ôtait aux rois les royaumes à cause de leurs justices et ne manque pas d'exercer sur eux la justice quand ils convertissent leur puissance en mauvais refuge ${ }^{84}$; «Et que tournant la tête devers les rebelles, <le roi > remplirait le Languedoc d'étonnement et avancerait beaucoup la réunion de tout le royaume et le rétablissement de son autorité en icelui, ce qui semble être le dessein de Dieu et le sujet de la bénédiction que S.M. en reçoit $\gg{ }^{85}$; «Cette heureuse guérison du roi semble vous obliger à le presser de sortir de là et rentrer dans son royaume, comme prenant ces accidents $[\ldots]$ comme avertissements que Dieu vous donne qu'il ne le veut pas en ce lieu-là $\gg{ }^{86}$.

Il arrive à Marillac d'invoquer la volonté de Dieu sans pour autant l'associer à un quelconque signe :

« Nous entendons ici force bruit des desseins du roi sur le passage en Italie et, je regarde tout cela en la confiance que j'ai que Dieu vous conduit. Il me semble que s'il plaît à Dieu que vous fassiez lever le siège de Casal, $[$... ] que c'est un effet glorieux et de grande réputation ${ }^{87}$; « Quand on considère que portant les armes du roi en Italie plus avant que ce qui est nécessaire $[\ldots]$ je ne sais pas si nous avons la bénédiction de Dieu en ces desseins-là » ${ }^{88} ;$ «Il me semblerait bien à propos de remplir toutes ces provinces de missionnaires, de récollets, capucins, pères de la doctrine, oratoriens, jésuites et autres [ ... ] c'est le fait des travaux et la fin et le but des desseins de Dieu ${ }^{89}$; « Cette personne si précieuse $<$ le roi $>$, et tant de besoins importants à la grandeur et prospérité de son royaume requièrent qu'il $[\ldots]$ y fasse les biens pour lesquels Dieu l'y a destiné $\gg^{90}$.

Dans ces cas, comment le roi connaît-il la volonté de Dieu? Le lecteur ne le sait pas et il sait encore moins comment Marillac la connaît. Ce qui est certain, c'est qu'à la différence de son homologue d'Aguesseau, qui sera garde des sceaux quelques années plus tard, Marillac n'a pas, ou seulement de façon accidentelle, adopté la méthode cartésienne d'accession à la volonté divine par la raison ${ }^{91}$. Ces passages montrent 
surtout que Marillac reconnaissait le roi comptable de son gouvernement devant Dieu. Si le pouvoir royal n'a pas déclaré la loi fondamentale la responsabilité du roi devant Dieu seul, comme le lui demandait le Tiers Etat lors des Etats Généraux de 1614, par crainte d'un schisme avec Rome, il reconnaissait, sous la plume de Marillac, la responsabilité de son gouvernement devant $\mathrm{Dieu}^{92}$.

\section{Conclusion}

Le Testament politique du cardinal de Richelieu justifie l'institution de principal ministre en prétendant qu'elle serait, au même titre que le roi, de droit divin. Bien que dans le système du ministériat, c'était le roi qui prenait les décisions en dernier lieu, cela ne justifiait pas pour autant, du point de vue des dévots, la délégation du gouvernement du royaume à un principal ministre. C'était contraire à l'esprit de la monarchie absolue selon lequel le roi devait gouverner seul, conformément à ses fondements, c'est-à-dire à la doctrine du droit divin et l'imagerie paternelle.

\section{Notes}

1. Ainsi qu'il est communément admis de l'employer, nous appliquerons aux dévots le terme de parti, pour désigner le cercle de la reine mère Marie de Médicis.

2. Le chercheur Donald A. Bailey, a souligné dans son travail sur la biographie de Michel de Marillac l'importance de ce personnage dans le règne de Louis XIII et la nécessité de le réhabiliter. Donald A. Bailey, « The family and early career of Michel de Marillac (1560-1632) », in Society and institutions in early modern France, éd. Mack P. Holt (Athens \& London : University of Georgia Press, 1991), p. 170-89; Bailey, « Power and piety: The religiosity of Michel de Marillac », Canadian journal of history/Annales canadiennes d'histoire 42.1 (printemps-été 2007), p. 1-24; Bailey, « Introduction », in Nicolas Lefèvre de Lezeau, La vie de Michel de Marillac (1560-1632). Garde des sceaux sous Louis XIII, éd. Donald A. Bailey, (Québec : Presses de l'Université Laval, 2007), p. xxv-lxx.

3. Ordonnance sur les plaintes des états assemblés à Paris en 1614, et de l'assemblée des notables réunis à Rouen, en 1617 et 1626, janvier 1629, Isambert, t. XVI, p. 223-344.

4. Michel de Marillac, Discours sur la manière de gouverner l'Estat, 10 juin 1630, A.A.E., mem. et doc. France, vol. 792, fo 207-214.

5. Michel de Marillac, Mémoire dressé par le garde des sceaux de Marillac principalement contre l'autorité $d u$ Parlement, manuscrit BnF fr. $75-49, \mathrm{f}^{\circ}{ }^{1-128}$; Marillac, Traité $d u$ Conseil du Roi, A.N., U 955 a, $\mathrm{f}^{\circ} 1-59$. Trois copies de ce traité sont conservées au département des manuscrits sous les cotes : $\mathrm{BnF}$ fr. $4582, \mathrm{BnF}$ fr, $4592, \mathrm{BnF}$ fr. 16218 ; Marillac, Traité des chanceliers et gardes des sceaux de France, A.N., U 948, f 2 r-4r. 
Plusieurs manuscrits de ce traité sont situés au département des manuscrits occidentaux sous les cotes : BnF fr. 18270, 18271, 18278 ; Marillac, Mémoire du 20 juillet 1630, A.A.E., Correspondance politique Sardaigne, vol. $12, \mathrm{f}^{\circ} 480-484$.

6. Pour ne citer que les plus importants : Donald A Bailey, Writers against the Cardinal : a study of the pamphlets which attacked the person and policies of the cardinal de Richelieu during the decade 1630-1640 (Ph. D. dissertation. Universty of Minnesota, 1973, 2 vols.) ; Seung Hwi Lim, La pensée politique des «bons catholiques 》 dans la première moitié $d u$ XVII ème siècle, thèse dactylographiée d'histoire, Paris IV, 1998 ; Georges Pagès, «Autour du grand orage Richelieu et Marillac : deux politiques », Revue historique 179 (1937), p. 63-97; Etienne Thuau, Raison d'État et pensée politique à l'époque de Richelieu (Paris : Albin Michel, 2000), pp. 103-52.

Cependant, l'historiographie n'a pas pris en compte les travaux de chercheurs nord-américains. J. Russel Major a démontré l'absolutisme de Marillac en se fondant sur son attitude alors qu'il était maître des requêtes, sa loyauté à la monarchie et son inclination pour le pouvoir centralisé. C'est ainsi que Marillac est considéré dans ces travaux comme «plus qu'un absolutiste », Russel J. Major, Representative gouvernement in Early Modern France (New Heaven, Ст : Yale University Press, 1980). Aujourd'hui, Donald A. Bailey explique, quant à lui, l'absolutisme de Marillac par sa rigueur personnelle et ses scrupules religieux, ce dernier ne cédant jamais au compromis quand il en allait du pouvoir absolu du roi. Dans ses travaux, absolutisme et religion sont donc réconciliés et permettent d'envisager le très dévot Marillac comme un partisan de la monarchie absolue. Cependant, ces études portent principalement sur le début de carrière de Michel de Marillac. Sur le point de la politique étrangère, qui a mené à la journée des Dupes, l'historiographie, dans son ensemble, présente toujours Marillac comme un opposant à la poursuite des guerres d'Italie, notamment parce qu'il était rebuté par l'entrée en guerre contre la catholique Espagne, Donald A. Bailey, « The family and early career of Michel de Marillac (1560-1632) », Society and institutions in early modern France, éd. Mack P. Holt , p. 170-89, et « Power and piety: The religiosity of Michel de Marillac $\gg$, p. 1-24.

7. Caroline Maillet-Rao, Mathieu de Morgues (1582-1670) et Michel de Marillac (15601632) : les dévots devant l'Histoire, thèse dactylographiée d'histoire du droit et des institutions, Tours, 2004, dont le texte a été entièrement remanié en vue de sa prochaine publication sous le titre : La pensée politique des dévots Mathieu de Morgues et Michel de Marillac. Une opposition au ministériat.

8. Mathieu de Morgues, Très-humble remontrance au Roi, dans Recueil des diverses pièces pour la défense de la reine mère du roi très-chrétien Louis XIII, 2 vols. (Paris : 1643, $1^{\text {ère }}$ éd. 1637), vol. 1, p. 38.

9. Mathieu de Morgues, Lumières pour l'histoire de France, in Recueil des diverses pièces pour la défense de la reine mère du roi très-chrétien Louis XIII, vol. 2, p. 261. Morgues écrit aussi plus loin : «Dupleix [ ... ] après avoir écrit que le Roi prêtait son nom à Monsieur de Luynes pour gouverner son Estat », p. 261-262. 
10. De Morgues, Lumières pour l'histoire de France, p. 287, 290, 305 et 357. Morgues écrit encore : «son jugement [de Dupleix] n'est pas meilleur, lorsqu'il donne l'autorité, au cardinal, qui ne doit appartenir qu'au Roi [ ... ] qu'il a voulu maintenir son crédit, qu'il appelle autorité », p. 322 ; ou : «Dupleix [ ... ] commence ainsi : Les rois sont mortels en France comme ailleurs mais pourtant la Monarchie Française n'est jamais Anarchie. Il est vrai, qu'une Monarchie n'est jamais Anarchie, c'est-à-dire un n'est jamais plusieurs, ni l'unité confusion $\gg$, p. 373.

11. Sur cette question, Arlette Jouanna, « Faveur et favoris : l'exemple des mignons d'Henri III », in Robert Sauzet (dir.), Henri III et son temps, Actes du colloque international du Centre de la Renaissance de Tours, Octobre 1989 (Paris : CNRS, 1992), p. 155, rappelle qu'aux $\mathrm{XVI}^{\mathrm{e}}$ et $\mathrm{XVII}$, les souverains français, anglais et espagnol se sont servis de personnages à qui ils ont délégué tout ou partie de leur autorité.

12. Richelieu, Testament politique, éd. Françoise Hildesheimer, (Paris : Société de l'histoire de France, 1995, $1^{\text {ère }}$ éd. 1643), I ère partie, chap. 8, p. 230. Cf. Jean-Pierre Brancourt, Le duc de saint-Simon et la monarchie, (Paris : Cujas, 1971), p. 75, relève que Richelieu fut le véritable théoricien du ministériat.

13. Thuau, Raison d'État et pensée politique à l'époque de Richelieu, p. 239.

14. Dans ce sens, Brancourt, Le Duc de Saint-Simon et la monarchie, pp. 80-81.

15. Richelieu, «Qui représente quel doit être le nombre des conseillers d'Etat et qu'entre eux il doit y en avoir un qui ait l'autorité supérieure», Testament politique, İ̀re partie, chap. 8 , section $6^{\mathrm{e}}$, p. 229.

16. En ce sens, Françoise Hildesheimer, « Le testament politique ou le règne de terrestre de la raison $\gg$, Annuaire bulletin de la société d'histoire de France (1994), pp. 24-27, explique que la raison dans le Testament politique, qui intervient explicitement pour définir l'homme et le relier à Dieu, tire sa source de Dieu. L'auteur se réfère également à la définition du dictionnaire de Trévoux définissant la raison comme la : « faculté naturelle dont Dieu a pourvu l'homme pour connaître la vérité $\gg$, qui permet de distinguer la définition rationnelle de celle de la Renaissance.

17. Par exemple : Orest Ranum, « Richelieu, l'histoire et les historiographes », in Roland Mousnier (dir.), Richelieu et la culture, Actes du colloque international en Sorbonne (Paris : CNRS, 1987), p. 127.

18. Richelieu, Testament politique, $\mathrm{I}^{\mathrm{r} r}$ partie, chap. 8 , section $6^{\mathrm{e}}, \mathrm{p} .232$.

19. Cf. Françoise Hildesheimer, « Les scrupules de Richelieu », Journal des savants (janv-juin 2000), p. 111.

20. Cardin Le Bret, De la souveraineté du Roi, (1632), chap. IX, p. 19.

21. Herman Weber, «Richelieu, théoricien politique », in Richelieu et la culture, p. 63.

22. «La souveraineté est la puissance absolue et perpétuelle d'une République [ ... ] Cette puissance est absolue et souveraine car elle n'a autre condition que la loi de Dieu et de nature ne commande [ ... ] En la monarchie, chacun en particulier et tout le peuple en corps doit jurer de garder les lois et faire serment de fidélité au monarque souverain, qui ne doit serment qu'à Dieu seul, duquel il tient le sceptre et la puissance $\gg$, Jean Bodin, Les six livres de la République (Paris : Fayard, 1986, 1 ère éd. 1576), vol. 
I, chap. 8, p. 179, 188 et 206. Dans le même sens, Françoise Hildesheimer, « Au cœur religieux du ministériat. La place de Dieu dans le Testament politique de Richelieu », Revue d'histoire de l'Eglise de France 84 (janv. 1998), p. 33.

23. De la même façon, Saint-Simon, Bossuet et Fénélon ont dénoncé le ministère de Mazarin et défendu, par-là même, l'indépendance du pouvoir royal. Sur cette question, voir Brancourt, Le duc de Saint-Simon et la monarchie, pp. 75-83. Aussi, en décidant d'exercer personnellement le pouvoir à la mort du favori d'Anne d'Autriche, Louis XIV a également manifesté son hostilité à l'égard du ministériat. Cf. Fanny Cosandey, Robert Descimon, L'absolutisme en France. Histoire et historiographie, (Paris : Seuil, collection Points histoire, 2002), p. 139.

24. Françoise Hildesheimer, « Les scrupules de Richelieu », p. 110 ; voir aussi sur la définition du ministériat: Hildesheimer, Richelieu, (Paris : Flammarion, 2004), p. 484.

25. Michel de Marillac, Discours sur la manière de gouverner l'Estat, Paris, Archives des Affaires étrangères (A.A.E.), mem. et doc. France, vol. 792, $\mathrm{f}^{\circ} 207 \mathrm{r}^{\circ}$.

26. Minute du Cardinal, Inventaire des Lettres du garde des Sceaux, A.A.E., mem. et doc. France, vol. $249, \mathrm{f}^{\circ} 163$.

27. « Le roi doit avoir soin qu'il y ait de bons évêques $[\ldots]$ qu' ils fassent leurs charges $[\ldots]$ Ce corps $[\ldots]$ mais $[\ldots]$ l'estime du roi vers cet ordre doit être avec circonspection pour les retenir s'ils passent plus avant qu'il ne faut », Marillac, Discours sur la manière de gouverner l'Estat, $\mathrm{f}^{\circ} 209 \mathrm{v}^{\circ}$.

28. «Il suffit de dire que ce sont personnes fort difficiles à gouverner, que rien ne peut contenter. Je dis ceux qui sont à la cour spécialement, ce ne sont qu'envies, jalousies, murmures et monopoles, mais il faut contenir les uns et les autres par diverses manières d'emplois, de gratifications [ ... ] Il faut être fort soigneux de savoir leurs menées, ceux qui ont créance vers eux, et avoir près d'eux des personnes confidentes pour pourvoir à tout », Marillac, Discours sur la manière de gouverner l'Estat, $\mathrm{f}^{\circ} 211 \mathrm{v}-212 \mathrm{r}$.

29. « Ils servent à maintenir les sujets en obéissance, faire reconnaître l'autorité du roi et tenir les grands en devoir. Le roi est obligé de les maintenir et soutenir [ ... ] Mais pour ce que la grandeur de leur puissance leur laisse quelquefois oublier qu'elle ait des bornes et qu'ils entreprennent au delà de leur pouvoir, même qu'ils s'ingèrent parfois à prendre connaissance du gouvernement de l'Etat, contrôler les rois en leur conduite, et les vouloir obliger à prendre des conseils à leur gré. Le roi doit [ ... ] user de sa puissance et leur faire voir jusqu'où va le pouvoir qu' ils ont de S.M., les contenir dedans. Et les empêcher de passer plus avant », Marillac, Discours sur la manière de gouverner l'Estat, $\mathrm{f}^{\circ} 212 \mathrm{r}$.

30. « Il y a quelques grandes villes principales auxquelles il y a plus d'affaires et sont plus mutines Il faut avoir grand soin de les maintenir en paix, n'enfreindre pas aisément leurs privilèges mais aussi faut prendre garde qu'elles ne s'élèvent pas et ne pas négliger les occasions de les faire avoir recours au roi pour plusieurs affaires, tenant le contrepoids entre le gouverneur et la ville pour s'en servir et les rendre tous dépendants de l'autorité du Roi », Marillac, Discours sur la manière de gouverner l'Estat, $\mathrm{f}^{\circ} 212 \mathrm{r}-213 \mathrm{v}$. 
31. «Il faut les supporter, les tenir comme les autres sujets et avoir compassion d'eux ayant toutefois l'esprit toujours ouvert à détruire la faction $\gg$, Marillac, Discours sur la manière de gouverner l'Estat, $\mathrm{f}^{\circ} 213 \mathrm{v}$.

32. Ainsi, de Morgues a employé le concept d'État dans un libelle dont le titre est évocateur à lui seul : La restauration de l'Estat. De cette manière, il a prétendu défendre l'autorité souveraine du roi, mise en danger par la rébellion des princes agacés par la trop grande influence du maréchal d'Ancre sur la régente. De la même façon, de Morgues a opposé la souveraineté de l'Etat aux parlementaires frondeurs, voir : Mathieu de Morgues, Bons avis sur plusieurs mauvais avis, (Paris : 1650), p. 3.

33. De Morgues, Lumières pour l'histoire de France, p. 261.

34. Tertullien (Carthage, c.155-220) : apologiste chrétien, Père de l'Église. Premier écrivain en latin de religion chrétienne, il est l'initiateur d'un vocabulaire théologique dans cette langue. Célèbre pour la violence de son style et la rigueur de ses prescriptions. Il finit par adopter les idées de Montan, prophète d'une secte chrétienne qui professait la supériorité du prophétisme sur la hiérarchie ecclésiastique, la venue imminente du paraclet, et la recherche du martyre. Le mouvement se répandit après 172 . Principales œuvres de Tertullien : Apologétiques (197), Contre Marcion (210).

35. Théodose $\mathrm{I}^{\mathrm{er}}$ le Grand (c.346-c.395) : empereur romain (379-395). Nommé Auguste par Gratien, il reçut le gouvernement de l'empire d'Orient à la place de Valens. Maxime s'étant fait proclamer empereur d'Occident, il le reconnut d'abord, tout en rétablissant sur le trône Valentinien II, puis marcha contre lui, le battit et le fit mettre à mort (388). Après la mort de Valentinien II, il écrasa l'usurpateur Eugène (394), Théodose resta le seul souverain, le dernier à avoir régné sur l'ensemble de l'empire. Il mourut quatre mois après avoir partagé l'empire entre ses deux fils. Sous son règne, le christianisme devint religion d'État ( 380$)$; l'empereur combattit avec force le paganisme, ordonna la fermeture des temples et interdit les sacrifices. Au cours d'un séjour à Milan, il se vit excommunié par saint Ambroise pour avoir ordonné le massacre de 7000 habitants de Thessalonique insurgée (390).

36. Marcien (396-457) : empereur d'Orient (450-457). Successeur de Théodose II dont il épousa la sœur Pulchérie, il refusa de payer le tribut à Attila et vint en aide à Valentinien III en Italie. Il combattit le monophysisme et réunit le concile de Chalcédoine (451). Il fut canonisé, ainsi que Pulchérie, par l'église grecque.

37. Tibère (42 av. J.-C.-37) : empereur romain (14-37). Fils de Livie, qui devait épouser Auguste en 38 av. J.-C.. Son père adoptif l'obligea à épouser Julie, sa fille, à l'instigation de Livie qui préparait l'accession de son fils au trône. Tous les autres prétendants à la succession étant morts, Auguste dut adopter Tibère en 9 av. J.-C., et en 14, il lui succéda aisément à 56 ans.

38. Néron (37-68) : empereur romain (54-68). Fils d'Agrippine la Jeune, il était par celle-ci le neveu de Caligula et l'arrière-petit-fils d'Auguste. Dévorée par l'ambition politique, Agrippine consacra toute son intelligence à faire accéder son fils au pouvoir ; elle le fit adopter par Claude qu'elle avait épousé en secondes noces ; et grâce aux manœuvres de Burrus, préfet du prétoire à la mort de Claude, Néron fut proclamé 
empereur par la garde prétorienne. Devenu despote déséquilibré, il fit tuer sa mère (59). Accusé d'être l'incendiaire du grand incendie de Rome (64), il détourna la responsabilité sur les chrétiens et déclencha contre eux une cruelle persécution.

39. Nous n'avons pas réussi à identifier de quel Basile il s'agissait.

40. Mathieu de Morgues, Le droit du roi sur des sujets Chrétiens à ceux de la religion prétendue réformée (Paris : 1622), p. 31-32; puis plus loin : « Cette règle doit vous faire connaître que vous n'avez aucun droit de vous opposer aux volontés du roi, si vous dites que vous êtes fondés sur un contrat avec lui. Je vous demanderai si l'écriture des hommes vous dispense des lois de l'Écriture Sainte si par les édits vous avez renoncé aux devoirs des chrétiens et si vous avez été transformés de sujets en associés », Mathieu de Morgues, Le droit du roi sur des sujets Chrétiens, p. 43.

41. Jean 19, 11 (Bible de Jérusalem).

42. De Morgues, Le droit du roi sur des sujets Chrétiens, p. 68-69.

43. Jacques Bénigne Bossuet, Cinquième avertissement aux protestants sur les lettres $d u$ ministre Jurieu contre l'histoire des variations (Caen : Centre de philosophie politique et juridique de l’Université de Caen, 1991, réédition de la première édition de 1690), pp. $380-488$.

44. Jacques Bénigne Bossuet, La politique tirée de l'Ecriture sainte (Genève : Droz, 1967,

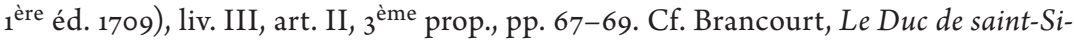
mon et la Monarchie, p. 35 ; Robert Pissere, Les idées politiques de Bossuet (Montpellier : Causse, 1943), pp. 33-45.

45. Cf. le libelle Défense du roi et de ses ministres.

46. Jacques Lelong, Bibliothèque historique de la France, contenant le catalogue des ouvrages imprimés et manuscrits, qui traitent de l'histoire de ce royaume, avec des notes critiques et historiques, (Paris : Fontette, 1768), précise que Jean Sirmond est le véritable auteur du libelle intitulé Défense du roi et de ses ministres.

47. Mathieu de Morgues, Vrais et bons avis d'un français fidèle. Sur les calomnies et blasphèmes du sieur des Montagnes, ou examen du libelle intitulé Défense du roi et de ses ministres, in Diverses pièces pour la défense de la Reine mère du roi très-chrestien Louis XIII, vol. 1, p. 132 ; dans le même sens, de Morgues conteste les propos de l'étatiste Scipion Dupleix (1569-1661) : « Il dit que : le roi ne rend compte de ses actions qu'à Dieu seul et, qu'il peut faire honorer ceux qui bon lui semble, pour des considérations telles qu'il lui plaît. Je m'assure que S.M [ ... ] ne demeurera pas d'accord avec de cette règle : celle de la justice ne vient jamais de la volonté et de la puissance absolue. Un flatteur semblable à Dupleix dit à Antigonus que toutes choses étaient honnêtes et justes pour les rois. Ce sage prince répondit que c'était une leçon pour les tyrans mais que, pour les bons rois, il n'y a rien d'honnête que ce qui est honnête, ni de juste que ce qui est juste $\gg$.

48. Mathieu de Morgues, Vrais et bons avis d'un français fidèle, p. 132.

49. Richelieu, Testament politique, p. 245 ; En revanche, Richelieu, Instruction du chrétien, leçon 11, cité par Hildesheimer, « Les scrupules de Richelieu », p. 113 ; Hildesheimer, « De la paix des armes à la paix de l'âme de Richelieu », in Paix des armes, paix des 
âmes, Actes du colloque international tenu au musée national du château de Pau et à l'Université de Pau et des Pays de l'Adour les 8, 9, 10, 110ctobre 1998, (Paris : Société Henri-IV-imprimerie nationale, 2000), p. 473, expose de façon explicite que « l'obligation de l'obéissance $[. .$. ] n'est pas seulement un impératif politique, mais aussi une nécessité d'ordre religieux ».

50. Richelieu, « Qui traite de la puissance du prince », Testament politique, II ${ }^{\text {ème }}$ partie, chap. IX, pp. 285-88.

51. Cf. Thuau, Raison d'État et pensée politique à l'époque de Richelieu, p. 245, qui parle de « religion de l'obéissance », tout en remarquant, au même endroit, que certains étatistes ont aussi «insisté sur le caractère religieux de l'obéissance ».

52. Cf. Jean de Viguerie, « Richelieu théologien », Richelieu et la culture, p. 37 : « La véritable opposition est d'ordre théologique et philosophique. Bérulle et Saint-Cyran ne croient pas à une raison pour eux corrompue. Richelieu fait confiance à la raison. C'est la différence fondamentale $\gg$.

53. Excepté peut-être à la fin de sa vie : «Comme le cœur des Rois est dans les mains de Dieu, aussi est bien leur esprit auquel il communique de grandes lumières pour les éclairer », de Morgues, Le prophète français, 1640, p. 7.

54. «Après que $<$ Richelieu $>$ a été le principal ministre de votre Estat ? Nous avons vu le royaume affligé de guerre, de peste, et de famine : les trois fléaux de Dieu », de Morgues, Très-humble remontrance au Roi, p. 6.

55. « Vous nous imposez que nous avons dit que le roi a triomphé de ses ennemis et a dompté ses sujets rebelles plutôt par miracle de Dieu, que par sa conduite [ ... ] Nous reconnaissons comme bons catholiques que Dieu a fait des miracles pour le roi et que le roi a fait des merveilles pour Dieu », de Morgues, Vrais et bons avis d'un français fidèle, p. 133. Morgues ajoute dans un autre libelle : « [... Dieu, lequel enverra bientôt le vrai et bon génie de la France, qui parlera ainsi au roi [ ... ] l’Ange tutélaire de la France pour vous apporter les lumières du ciel. Il vous avertit que sa sainte providence qui vous aime, qui a imprimé dans le cœur de vos sujets le respect qu' ils vous portent, qui a jeté la terreur dans l'âme de vos ennemis et qui vous a donné des avantages sur eux, est sur le point de retirer ses grâces », Le génie démasqué, Diverses pièces pour la défense de la Reine mère du Roi très-chrétien Louys XIII, vol. 1, p. 451-52; «Dans le discours que vous appelez Coup d'Estat, vous dites [ ... ] Par moi les rois règnent. Cela est autant éloigné de la vérité comme de la modestie. Il y aurait même quelque impiété de croire que la providence divine n'eut point d'autres moyens que ceux que vous pouvez fournir pour conserver le roi et son Estat $[\ldots]$ vos actions $[\ldots]$ ne peuvent produire que toutes sortes de malheurs ; si vos desseins ne sont bientôt arrêtés par la puissante main de Dieu $\gg$, Mathieu de Morgues, Charitable remontrance de caton chrétien au cardinal de Richelieu, dans Diverses pièces pour la défense de la Reine mère du Roi très-chrétien Louis XIII, vol. 1, p. 302. Dans un autre libelle, Morgues poursuit : « Cet infâme flatteur [Sirmond] dit aussi : le cardinal fait régner le roi [ ... ] Il le fait régner, il est donc Dieu qui a dit : Je fais régner les Rois », de Morgues, Le génie démasqué, p. 447. 
56. Dans ce sens, voir Hildesheimer, « Le testament politique de Richelieu ou le règne terrestre de la raison $\gg$, p. 24 ; Hildesheimer, Au cœur religieux du ministériat, p. 36 ; Hildesheimer, Relectures de Richelieu (Paris : Publisud, 2000), p.73 ; Hildesheimer, Richelieu, pp. 329-40.

57. Cf. de Viguerie, « Richelieu théologien », p. 31.

58. Cf. Weber, «Richelieu, théoricien politique », p. 59.

59. Pour de Viguerie, « Richelieu théologien », p. 34, « la raison du Testament politique et la raison du Traité de la perfection sont bien la même raison $\gg$. Sur la notion de raison dans les écrits théologiques de Richelieu, voir aussi : Stéphane-Marie Morgain, « Présentation », in Richelieu, Traité de la perfection du chrétien, Euvres théologiques, texte établi et introduit par Françoise Hildesheimer et Stéphane-Marie Morgain (Paris : Honoré Champion, 2002), pp. 83-84.

6o. Richelieu, «Qui montre que la raison doit être la règle et la conduite d'un Estat », Testament politique, p. 245 ; et encore : « Mais c'est une chose si connue d'un chacun par sa propre raison qu'il ne tire pas son être de lui-même, mais qu'il a un Dieu pour créateur et, par conséquent, pour directeur, qu'il n'y a personne qui ne sente que la nature a imprimé cette vérité dans son cœur avec des caractères qui ne peuvent s'effacer $[\ldots$. La raison doit être le flambeau qui éclaire les princes en leur conduite et en celle de leur Etat $[. .$.$] Un prince doit surtout éviter d'agir selon un$ tel principe < la passion $>$ qui le rendrait d'autant plus odieux qu'il est directement contraire à celui qui distingue l'homme d'avec les animaux »; Richelieu, Testament politique, p. 241-42 et 246; cf. Roland Mousnier, «Conclusion générale », Richelieu et la culture, p. 225 ; Emmanuel Le Roy Ladurie, L’Ancien Régime (Paris : Hachette, collection Histoire de France, 2000, 1 ère éd. 1991), p. 71, souligne que la raison est le moyen par lequel Dieu « inspire ou souffle » les « opinions valables » au Prince ; Jean de Viguerie, «Richelieu théologien », p. 34, relève que chez Richelieu la raison « enseigne les voies de la justice et à ne rien vouloir que ce qui est raisonnable et juste » et Françoise Hildesheimer, « Richelieu ou la rhétorique de la raison », in Bernard Barbiche, Jean-Pierre Poussou et Alain Tallon (dir.), Pouvoirs, contestations et comportements dans l'Europe moderne. Mélanges en l'honneur d'Yves-Marie Bercé (Paris : Presses Universitaires de France, 2005), p. 227-45.

61. Cf. Isabelle Storez, Le chancelier Henri François d'Aguesseau. Monarchiste et libéral (Paris : Publisud, 1996), p. 432.

62. Storez, Le chancelier Henri François d'Aguesseau, pp. 431-67.

63. René Descartes, Méditations métaphysiques (Paris : Vrin, 1976, 1 ère éd. 1647), p. 3940.

64. Michel de Marillac, Discours sur la manière de gouverner l'Etat, $\mathrm{f}^{\circ} 207 \mathrm{r}$.

65. Harangue du sieur garde des Sceaux, Dijon, 28 février 1630, Mercure français, t. XVI, p. 157.

66. Michel de Marillac, Harangue de Monsieur le garde des Sceaux, faite aux Etats de Bretagne tenus à Nantes, le Roy y séant, 11 juillet 1626, bibliothèque royale, p. 14 .

67. Michel de Marillac, Discours sur la manière de gouverner l'Etat, $\mathrm{f}^{\circ} 207 \mathrm{r}$. 
68. Cf, Yves-Marie Bercé, Le roi père, in Les monarchies (Paris : Presses Universitaires de France, 1997), pp. 231-39.

69. Plutôt chambre d'enregistrement triée sur le volet plutôt que véritables États généraux, les députés avaient été plus nommés par les autorités locales que véritablement élus. À raison de deux députés par bonne ville, en général un noble et un bourgeois, il régnait dans cette réunion une atmosphère anormalement calme et consensuelle. Éminemment méfiant à l'égard de cette institution, Louis XII réunit ses représentants pour des objectifs bien précis, en montrant bien qu'il ne s'agit pas pour eux de faire entendre des doléances, mais de plébisciter sa politique. L’assemblée de Tours devait démontrer l'approbation de l'opinion publique tout entière au nouveau projet de mariage de la princesse Claude. Sur Louis XII, cf. : Didier Le Fur, Louis XII, 1498-1515, un autre César (Paris : Perrin, 2001) et Bernard Quillet, Louis XII, Père du Peuple (Paris : Fayard, 1986).

70. Thomas Bricot : personnage considérable, Chanoine de Notre-Dame, professeur de philosophie à l'université de Paris et spécialiste d'Aristote.

71. Récit de ce qui s'est passé lors de la remonstrance faite au roi Louis XII par les Etats du royaume, pour l'engager à consentir au mariage de Madame Claude de France avec Monseigneur François, duc de Valois, in Lettres de Louis XII (Bruxelles : Godefroy, 1712), vol. I, p. 43.

72. Michel de Marillac, Discours sur la manière de gouverner l'Estat, $\mathrm{f}^{\circ} 207 \mathrm{r}$.

73. Marillac, Discours sur la manière de gouverner l'Estat, $\mathrm{f}^{\circ} 209 \mathrm{v}$.

74. Marillac, Discours sur la manière de gouverner l'Estat, $\mathrm{f}^{\circ} 210 \mathrm{r}$.

75. Marillac, Discours sur la manière de gouverner l'Estat, $\mathrm{f}^{\circ} 212 \mathrm{v}$.

76. Marillac, Discours sur la manière de gouverner l'Estat, $\mathrm{f}^{\circ} 211 \mathrm{v}$.

77. Déclaration qui défend aux ministres de la religion protestante qui sont étrangers, d'exercer en France aucune fonction, à ceux qui sont nés en France, d'en sortir sans permission du roi, avril 1627, Isambert, t. XVI, p. 202.

78. Refus d'enregistrer l'ordonnance de 1629.

79. Ordonnance sur les plaintes des états assemblés à Paris en 1614, p. 223-344.

8o. Ordonnance sur les plaintes des états assemblés à Paris en 1614, p. 226.

81. Le commandement divin de l'obéissance au roi s'applique d'ailleurs à tous les sujets sans exception. Marillac affirme à plusieurs reprises obéir lui-aussi aux commandements du roi comme s'ils venaient de Dieu : « Tous les commandements du Roi me sont saints » ; Michel de Marillac au Cardinal de Richelieu, Lyon, 25 juin 1630, A.A.E., mem. et doc. France, vol. 795 bis, $\mathrm{f}^{\circ} 184$, publié par Pierre Grillon, Les papiers de Richelieu (Paris : A. Pedone, 1975-1985), vol. 5, p. 340 ; ou, dans une autre lettre, « Nous attendons les commandements de S.M. pratiquant une obéissance fort religieuse »; Michel de Marillac à Bouthiller, Grenoble, $1^{\text {er }}$ août 1630, A.A.E., mem. et doc. France, vol. 795 bis, $\mathrm{f}^{\circ} 243$.

82. Marillac, Mémoire contre l'autorité du Parlement, $\mathrm{f}^{\circ} 96$.

83. Marillac, Discours sur la manière de gouverner l'Estat, $\mathrm{f}^{\circ} 212 \mathrm{v}$.

84. Marillac, Discours sur la manière de gouverner l'Estat, $\mathrm{f}^{\circ} 128$. 
85. Ibid., p 104 ; ou ailleurs : « Le but des desseins de Dieu par lequel il a fait des merveilles dont vous avez été très digne $\gg$, Michel de Marillac au Cardinal de Richelieu, 16 août $1629, f^{\circ} 97$.

86. Michel de Marillac au Cardinal de Richelieu, Grenoble, 22 juillet 1630, A.A.E., correspondance politique Sardaigne, vol. 12 , f ${ }^{\circ}$ O3-504, publié par Pierre Grillon, Les papiers de Richelieu, t. V, p. 419.

87. Michel de Marillac au Cardinal de Richelieu, Paris, 15 février 1629, A.A.E., mem. et doc. France, vol. 793 , f ${ }^{\circ} 74-75$, publié par Pierre Grillon, Les papiers de Richelieu, vol. 4, p. 103.

88. Marillac au Cardinal de Richelieu, Paris, 15 février 1629, in Grillon, Les papiers de Richelieu, vol. 4, p. 104.

89. Michel de Marillac au Cardinal de Richelieu, Paris, 16 août 1629, A.A.E., mem. et doc. France, vol. 794, $\mathrm{f}^{\circ} 97$.

90. Michel de Marillac au Cardinal de Richelieu, Grenoble, 20 juillet 1630, A.A.E., correspondance politique Sardaigne, vol. 12, f $\mathrm{f}_{484}$ (Mémoire publié partiellement par Pierre Grillon, Les papiers de Richelieu, vol. 5, p. 428-431).

91. Sur cette question, voir : Isabelle Storez, Le chancelier Henri François D'Aguesseau, pp. $427-32$.

92. Cette conclusion se base surtout sur les extraits tirés du Discours sur la manière de gouverner l'Estat. 